\title{
Influence of Herbal Complexes Containing Licorice on Potassium Levels: A Retrospective Study
}

\author{
WooSang Jung, ${ }^{1}$ SeungWon Kwon, ${ }^{1}$ JinWook Im, ${ }^{1}$ SeongUk Park, ${ }^{2}$ SangKwan Moon, \\ JungMi Park, ${ }^{2}$ ChangNam Ko, ${ }^{2}$ and KiHo Cho ${ }^{1}$ \\ ${ }^{1}$ Department of Cardiovascular and Neurologic Diseases, Kyung Hee University Oriental Medicine Hospital, \\ 1 Hoegi-dong, Dongdaemun-gu, Seoul 130-702, Republic of Korea \\ ${ }^{2}$ Department of Cardiovascular and Neurologic Diseases, Kyung Hee University Hospital at Gangdong, \\ Seoul 134-727, Republic of Korea \\ Correspondence should be addressed to SeungWon Kwon; kkokkottung@hanmail.net
}

Received 11 February 2014; Revised 1 May 2014; Accepted 19 May 2014; Published 19 June 2014

Academic Editor: Paul Posadzki

Copyright (C) 2014 WooSang Jung et al. This is an open access article distributed under the Creative Commons Attribution License, which permits unrestricted use, distribution, and reproduction in any medium, provided the original work is properly cited.

\begin{abstract}
To observe the influence of these complexes on potassium levels in a clinical setting, we investigated the influence of herbal complexes containing licorice on potassium levels. We retrospectively examined the medical records of patients treated with herbal complexes containing licorice from January 1, 2010, to December 31, 2010. We recorded the changes in the levels of potassium, creatinine, and blood urea nitrogen and examined the differences between before and after herbal complexes intake using a paired $t$-test. In addition, we investigated the prevalence of hypokalemia among these patients and reviewed such patients. We identified 360 patients who did not show significant changes in the levels of potassium and creatinine $(P=0.815,0.289)$. We observed hypokalemia in 6 patients. However, in 5 patients, the hypokalemia did not appear to be related to the licorice. Thus, we could suggest that herbal complexes containing licorice do not significantly influence the potassium levels in routine clinical herbal therapies. However, we propose that follow-up examination for potassium levels is required to prevent any unpredictable side effects of administration of licorice in routine herbal medicine care.
\end{abstract}

\section{Introduction}

Licorice has been used as a medicinal agent for patients of all ages and in East Asian countries. Licorice has been used for the treatment of peptic ulcer, as an anti-inflammatory agent, expectorant, drink, candy, and sweetener $[1,2]$. In particular, in Korean medicine, licorice has been used in many herbal prescriptions for the treatment of sore throat, cough, and wounds and for neutralizing the poisonous effects of other herbs such as Aconiti Ciliare Tuber, Ephedrae Herba, or Rhei Rhizoma. Therefore, East Asian people who prefer taking herbal complexes for treating their various symptoms frequently consume licorice.

Licorice was reported to cause hypokalemia for the first time in 1950 [3]. Subsequently, many studies have shown that licorice can induce hypokalemia, muscle weakness, and hypertension [1, 4-17]. In serious situation, licorice consumptions can cause rhabdomyolysis with generalized muscle aches or weakness, hematuria, hypokalemia, and renal dysfunction [8-10]. These studies suggest that long-term use and overdose of licorice can lead to severe hypokalemia and can be life threatening. However, previous studies only included case reports.

In the present study, we investigated the influence of herbal complexes containing licorice on potassium levels in 360 cases. We aimed to observe the influence of such herbal complexes on potassium levels in a clinical situation.

\section{Methods}

We examined the medical records of patients who were treated with herbal complexes containing licorice from January 1 , 
2010, to December 31, 2010, at the Kyung Hee University Oriental Medicine Hospital, Seoul, Korea. From these patients, we selected subjects whose potassium levels were stable before the administration of herbal medication and whose potassium levels were in the normal range. Thus, we excluded patients with hypokalemia or hyperkalemia at the starting point of the study. Further, we selected subjects whose herbal medication and existing medications such as antiplatelet agents, antihypertensive agents, antidiabetic agents, statins, or nonsteroidal anti-inflammatory drugs had not changed during followup. If the composition of the herbal complexes or the dose of licorice or other medications changed, we assumed that followup was completed. We recorded the patients' characteristics, reasons for using herbal complexes, dose of licorice in each patient, duration of herbal medication intake, and changes in the levels of potassium, blood urea nitrogen (BUN), and creatinine. To measure the differences in potassium levels during administration of herbal medicines containing licorice (before and after herbal medicine intake), we used a paired $t$-test. Statistical analysis was performed using SPSS for Windows, version 10.0 (SPSS Inc., Chicago, IL, USA). Subsequently, we reviewed the cases of patients who showed hypokalemia during followup to assess the probable causes that affected the patients' potassium levels. When we reviewed the cases revealed hypokalemia, we used the Naranjo scale to estimate the influence of medication objectively.

\section{Results}

3.1. Patients' Characteristics. We identified 360 subjects fulfilling our criteria. The average dose of licorice was $8.7 \pm$ $4.1 \mathrm{~g} /$ day (average \pm standard deviation [SD]). Further, the average intake duration was $18.9 \pm 19$ days. The frequency of usage of herbal medicines was the highest for cerebral infarction $(62.4 \%)$. Other characteristics of the patients at baseline are listed in Table 1.

3.2. Changes in the Levels of Potassium, BUN, and Creatinine. Although the potassium level decreased during follow-up examination, this decrease was not statistically significant (potassium levels before versus after, $4.00 \pm 0.4$ versus $3.99 \pm$ $0.4 ; P=0.815)$. BUN and creatinine levels also decreased. The levels of BUN showed a statistically significant decrease (before versus after, $13.91 \pm 6.4$ versus $12.64 \pm 4.9 ; P<$ 0.001 ) (Table 2). However, all values were within the normal range (potassium 3.5 5.0 mEq/L; BUN 10 26 mg/dL; and creatinine $0.6 \sim 1.2 \mathrm{mg} / \mathrm{dL}$ ).

3.3. Patients with Hypokalemia. Six patients (1.7\%) had hy-pokalemia during the follow-up period (Table 3 ). The remaining 354 patients (98.3\%) had normal levels of potassium during the intake of the herbal complexes.

The cases of patients with hypokalemia are as follows (Table 4).

Case 1 was a 78-year-old woman who presented dysarthria and right upper and lower extremities weakness with a history of cerebral infarction. Over a 22-day period, she
TABLE 1: Baseline characteristics $(n=360)$.

\begin{tabular}{|c|c|c|}
\hline Characteristics & Frequency & Percentage \% \\
\hline Male/female & $182 / 178$ & $50.6 / 49.4$ \\
\hline Age, years $($ Mean \pm SD $)$ & $65.1 \pm 13.9$ & \\
\hline \multicolumn{3}{|l|}{ Causes of herbal medicine intake } \\
\hline Cerebral infarction & 224 & 62.4 \\
\hline Intracerebral hemorrhage & 88 & 24.5 \\
\hline Brain tumor & 2 & 0.6 \\
\hline Moyamoya disease & 4 & \\
\hline Parkinson disease & 4 & 1.1 \\
\hline Parkinsonism & 5 & 1.4 \\
\hline Anoxic brain damage & 23 & 6.4 \\
\hline Tension-type headache & 1 & 0.3 \\
\hline Transverse myelitis & 1 & 0.3 \\
\hline C-HNP & 6 & 1.7 \\
\hline T-HNP & 1 & 0.3 \\
\hline L-HNP & 7 & 1.9 \\
\hline Spinal stenosis & 4 & 1.1 \\
\hline Facial palsy & 11 & 3.1 \\
\hline Lung cancer & 3 & 0.8 \\
\hline Esophagus cancer & 1 & 0.3 \\
\hline Colon cancer & 1 & 0.3 \\
\hline Liver cancer & 3 & 0.8 \\
\hline Kidney cancer & 1 & 0.3 \\
\hline Gastric cancer & 1 & 0.3 \\
\hline Gall bladder cancer & 1 & 0.3 \\
\hline GERD & 1 & 0.3 \\
\hline DVT & 1 & 0.3 \\
\hline Osteoarthritis & 3 & 0.8 \\
\hline CRPS & 2 & 0.6 \\
\hline $\begin{array}{l}\text { Licorice dose (per day) in g (Mean } \pm \\
\text { SD) }\end{array}$ & $8.7 \pm 4.1$ & \\
\hline $\begin{array}{l}\text { Duration of administration in days } \\
\text { (Mean } \pm \text { SD) }\end{array}$ & $18.9 \pm 19$ & \\
\hline
\end{tabular}

GERD: gastroesophageal reflux disease; DVT: deep vein thrombosis; CRPS: complex regional pain syndrome type; C-HNP: cervical herniated nucleus pulposus; T-HNP: thoracic cervical herniated nucleus pulposus; L-HNP: lumbar herniated nucleus pulposus.

received aspirin to prevent the recurrence of stroke and the herbal complex Shipyukmiyouki-eum to treat sequelae after cerebral infarction. During treatment with these agents, her potassium level decreased from $4.0 \mathrm{mEq} / \mathrm{L}$ to $2.5 \mathrm{mEq} / \mathrm{L}$.

Case 2 was a 60 -year-old woman diagnosed with cerebral hemorrhage, hypertension, and urinary tract infection. The patient was referred by the neurosurgery department to be treated with herbal complexes and acupuncture therapy to treat her left side paralysis and left shoulder pain caused by cerebral hemorrhage. She started treatment with antibiotics for the treatment of urinary tract infection that occurred during rehabilitation 5 days before administration of the herbal complex Seogyung-tang. Over a 6-day period, she received ciprofloxacin, ceftriaxone sodium, oxiracetam, and 
TABLE 2: Measurement of the levels of potassium, blood urea nitrogen, and creatinine $(n=360)$.

\begin{tabular}{lccc}
\hline Laboratory findings & First & Second & $P$ value \\
\hline Potassium level $(\mathrm{mEq} / \mathrm{L})$ & $4.00 \pm 0.4$ & $3.99 \pm 0.4$ & 0.815 \\
Blood urea nitrogen level $(\mathrm{mg} / \mathrm{dL})$ & $13.91 \pm 6.4$ & $12.64 \pm 4.9$ & $<0.001$ \\
Creatinine level $(\mathrm{mg} / \mathrm{dL})$ & $1.04 \pm 7.4$ & $0.6 \pm 0.3$ & 0.289 \\
\hline
\end{tabular}

Values are average (standard deviation [SD]) and were compared using paired $t$-test.

TABLE 3: Incidence of hypokalemia $(n=360)$.

\begin{tabular}{lc}
\hline & Numbers (\%) \\
\hline Hypokalemia & $6(1.7)$ \\
Nonhypokalemia & $354(98.3)$ \\
\hline
\end{tabular}

Hypokalemia in this table indicates that potassium levels (at end point) are below $3.0 \mathrm{mEq} / \mathrm{L}$.

the herbal complex Seogyung-tang. During this series of treatments, her potassium level decreased from $3.5 \mathrm{mEq} / \mathrm{L}$ to $2.5 \mathrm{mEq} / \mathrm{L}$.

Case 3 was a 56-year-old man diagnosed with cerebral hemorrhage and hypertension. The patient was admitted to treat hemiplegia and insomnia caused by cerebral hemorrhage. He had used lercanidipine $\mathrm{HCl}$, atenolol, hydrochlorothiazide, and losartan potassium to control his hypertension and to prevent the recurrence of cerebral hemorrhage. After 14 days of onset of cerebral hemorrhage, he started treatment with Gamiondamg-tang to relieve insomnia over a 29-day period. During this treatment, his potassium level decreased from $3.9 \mathrm{mEq} / \mathrm{L}$ to $2.8 \mathrm{mEq} / \mathrm{L}$.

Case 4 was a 77-year-old woman with cerebral infarction, hypertension, and diabetes. She was referred by the neurosurgery department for treatment of insomnia, palpitation, and hemiparesis caused by stroke. She had taken $S$-amlodipine, clopidogrel, calcium carbonate, pravastatin sodium, donepezil $\mathrm{HCl}$, metformin $\mathrm{HCl}$, and nicergoline to control the risk factors of stroke (hypertension, dyslipidemia, and diabetes mellitus). Over a 20-day period, she received the herbal complex Gwibi-tang to treat insomnia, palpitation, and sporadic diarrhea, which may be caused by metformin $\mathrm{HCl}$. During this treatment, her potassium level decreased from $3.6 \mathrm{mEq} / \mathrm{L}$ to $2.6 \mathrm{mEq} / \mathrm{L}$.

Case 5 was an 89-year-old woman with hypertension and coronary artery disease. After coronary angioplasty, she was referred by the cardiology department to treat the residual fatigue. At the time of admission, she had taken fluvastatin sodium, aspirin, nifedipine, atenolol, hydrochlorothiazide, tramadol $\mathrm{HCl}$, acetaminophen, teprenone, and alfacalcidol. Over an 11-day period, she took the herbal complex Bojungikki-tang to treat her symptoms of fatigue. During this series of treatments, her potassium level decreased from $3.0 \mathrm{mEq} / \mathrm{L}$ to $2.2 \mathrm{mEq} / \mathrm{L}$.

Case 6 was a 72-year-old woman diagnosed with cerebral infarction and hypertension, and she was referred by the neurosurgery department to treat sequelae of stroke after craniectomy. She had used quetiapine to treat delirium, gabapentin to treat neuralgia, aspirin to prevent stroke recurrence, and sodium valproate, dimethicone, hemicellulase, ox bile extract, and pancreatin. Over an 8-day period, she used the herbal complex Banhasashim-tang to treat her dyspepsia and abdominal discomfort. During this series of treatments, her potassium level decreased from $3.7 \mathrm{mEq} / \mathrm{L}$ to $2.8 \mathrm{mEq} / \mathrm{L}$.

However, symptoms such as muscle weakness, hypertension, and renal failure were not observed in any of these 6 patients.

\section{Discussion}

In this study, we found no significant change in the potassium levels during the administration of herbal complexes containing licorice in 360 patients for $18.9 \pm 19$ days. Follow-up examination indicated that the potassium level decreased, but this decrease was not statistically significant $(P=0.815)$. The potassium levels were normal in $98.3 \%$ patients during the intake of herbal complexes. Only $1.7 \%$ patients had hypokalemia during followup. The average dose of licorice was $8.7 \pm 4.1 \mathrm{~g} / \mathrm{day}$, and the average administration duration was $18.9 \pm 19$ days. Therefore, we suggested that administration of herbal complexes containing licorice could be safe when the dose of licorice was less than $8.7 \pm 4.1 \mathrm{~g} /$ day and the duration of administration was less than $18.9 \pm 19$ days.

We observed 6 patients (1.7\%) who developed hypokalemia after treatment with herbal medicine complexes containing licorice. However, we believed that the development of hypokalemia could not be attributed only to licorice in almost all patients (5 patients). We believe that concomitant administration of western medicines such as antihypertensive and antidiabetic agents and the medical condition of patients affected the development of hypokalemia.

Cases 3 and 5 took the antihypertensive agent hydrochlorothiazide. Diuretics such as thiazides are common cause of drug-induced hypokalemia [18]. Therefore, we assumed that long-term intake of hydrochlorothiazide can be a cause of hypokalemia in cases 3 and 5. Case 4 used metformin $\mathrm{HCl}$ to control her blood glucose levels. Before administration of the herbal medicine Gwibi-tang, the patient had sporadic diarrhea and soft stool. A previous case report indicated that a 57-year-old Caucasian male had hypokalemia, hypocalcemia, and hypomagnesemia induced by long-term administration of metformin $\mathrm{HCl}$ [19]. Therefore, we believe that longterm intake of metformin $\mathrm{HCl}$ can be attributed to the development of hypokalemia in case 4 . Case 6 had used sodium valproate since craniectomy. A previous case report 


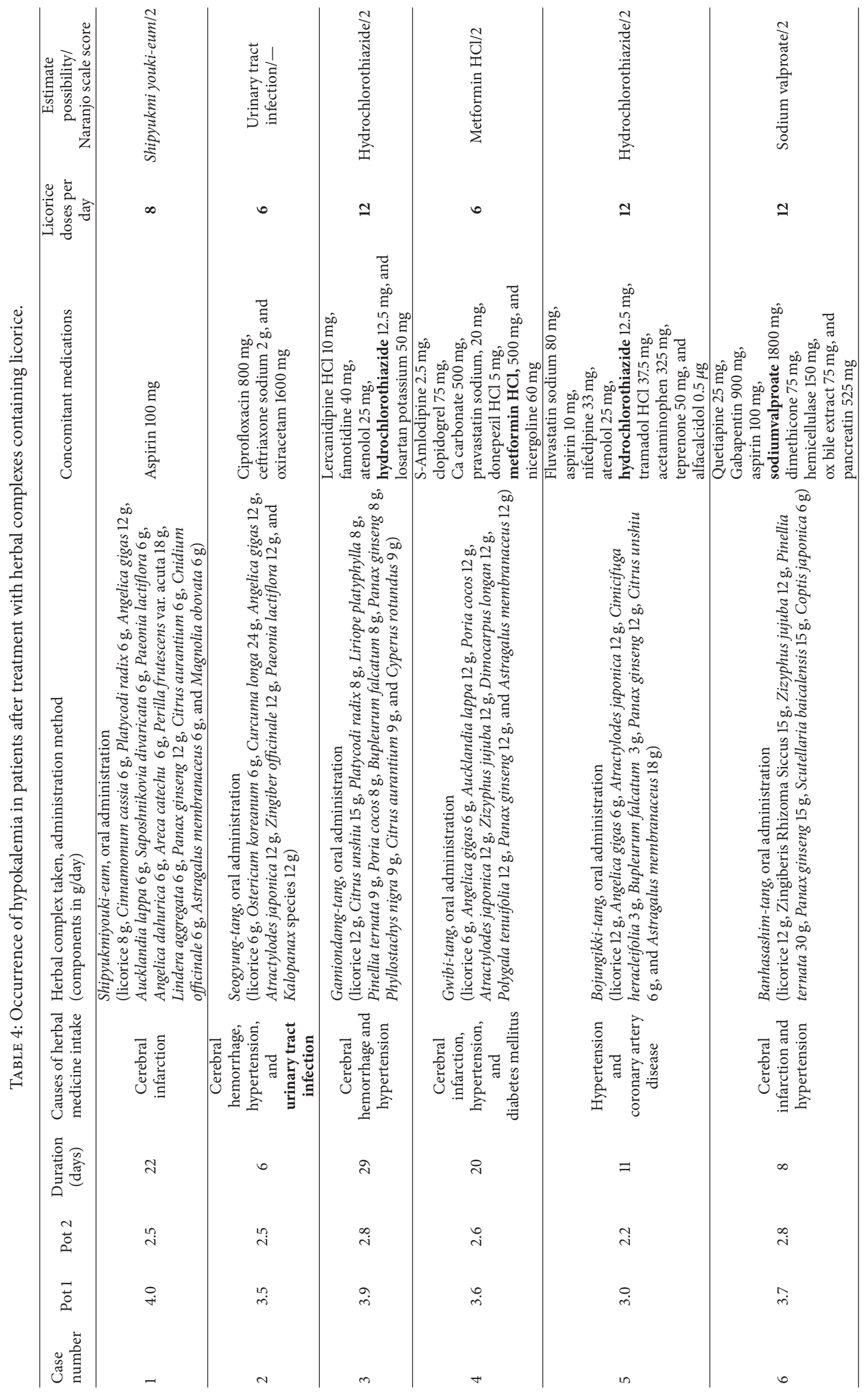


[20] indicated that a 14-year-old Japanese patient sometimes had fever and hypokalemia after intake of sodium valproate for 6 years. Therefore, we believe that consumption of sodium valproate can be a cause of hypokalemia in Case 6. In Case 2 , fever caused by urinary tract infection may be attributed to the development of hypokalemia. Moreover, a previous study showed that the prevalence of hypokalemia in patients hospitalized for infection at an institution was 23\% [21]. Thus, our results show that herbal complexes containing licorice cannot be a sole reason for the development of hypokalemia in these 5 patients.

Among the 360 patients, 1 patient (Case 1) had definite licorice-induced hypokalemia. In the case of this patient, no reasons other than the intake of Shipyukmiyoukieum (licorice $8 \mathrm{~g}$ ) could be attributed to the development of hypokalemia. This patient only used aspirin during Shipyukmiyouki-eum intake. No previous study to date suggests that aspirin may cause hypokalemia. Therefore, Shipyukmiyouki-eum can be a reason for hypokalemia.

Our study has the following limitations. We did not measure the serum levels of aldosterone, rennin, cortisol, and adrenocorticotropic hormone (ACTH). Therefore, we could not observe the influence of herbal complexes containing licorice on the endocrine system. Furthermore, because of retrospective nature of the study, we did not measure the amounts of glycyrrhetic acid which is the active component in licorice and duration of licorice contained herbal complexes intake and licorice dosage in each patients were not equal.

However, we believe that our findings can be a guide for safe use of licorice. We examined the average duration (18.9 \pm 19 days) and dose of licorice $(8.7 \pm 4.1 \mathrm{~g}$ /day) use in these 360 patients. Our results revealed that severe hypokalemia did not occur during administration of herbal complexes containing licorice. Moreover, our study reflects the clinical circumstances better than those reported in previous studies. Doctors in Korea, Japan, and China who use herbal medicines to treat patients usually prescribe many types of herbs simultaneously. All patients in the present study consumed herbal complexes containing licorice. Previous studies have reported the cases of patients consuming only licorice $[1,4-$ 17].

Our data suggest that administration of herbal complexes containing licorice does not affect the potassium levels when the dose of licorice is less than $8.7 \pm 4.1 \mathrm{~g}$ /day and duration of administration is less than $18.9 \pm 19$ days. However, we think that hypokalemia in patients who use licorice can be made by interaction between licorice and drug which might cause electrolyte disturbances and renal function. Thus, we can assume that followup of potassium levels is required to prevent any unpredictable side effects of longterm administration of herbal complexes containing licorice in elderly people, especially, who used herbal medication with western medication.

Furthermore, we suggest that well designed prospective study, with standard administration (dosages of licorice and intake duration) and documented amount of glycyrrhetic acid, is necessary to solve the question of possible licorice induced side effects.

\section{Conclusion}

The present study assessed the influence of herbal complexes containing licorice on potassium levels. This study was conducted as a retrospective, chart review study. The results of this study are as follows.

(1) Three hundred and sixty patients did not show significant changes in the levels of potassium and creatinine $(P=0.815,0.289)$.

(2) The average dose of licorice was $8.7 \pm 4.1 \mathrm{~g} /$ day. Further, the average intake duration was $18.9 \pm 19$ days.

(3) Six patients revealed hypokalemia. However, in 5 patients, the hypokalemia did not appear to be related to the herbal complex therapy containing licorice.

Therefore, we could suggest that administration of herbal complexes containing licorice does not affect the potassium levels at low dose and for a short period of time. However, we suggest that followup of potassium levels is needed to prevent any unpredictable side effects of administration of herbal complexes containing licorice.

\section{Disclosure}

No financial support was received during the present study.

\section{Conflict of Interests}

The authors declare that they have no conflict of interests.

\section{References}

[1] S. Shintani, H. Murase, H. Tsukagoshi, and T. Shiigai, "Glycyrrhizin (licorice)-induced hypokalemic myopathy: report of 2 cases and review of the literature," European Neurology, vol. 32, no. 1, pp. 44-51, 1992.

[2] B. R. Walker and C. R. W. Edwards, "Licorice-induced hypertension and syndromes of apparent mineralocorticoid excess," Endocrinology and Metabolism Clinics of North America, vol. 23, no. 2, pp. 359-377, 1994.

[3] F. E. Cayley, "Potassium deficiency in p-aminosalicylic acid therapy: cardiac and paralytic effects," The Lancet, vol. 255, no. 6602, pp. 447-448, 1950.

[4] J. W. Conn, D. R. Rovner, and E. L. Cohen, "Licoriceinduced pseudoaldosteronism. Hypertension, hypokalemia, aldosteronopenia, and suppressed plasma renin activity," Journal of the American Medical Association, vol. 205, no. 7, pp. 492496, 1968.

[5] S. Cho, B. Lim, H. Cho et al., "Licorice Induced Hypokalemia," Korean Journal of Nephrology, vol. 20, pp. 1021-1025, 2001.

[6] H. I. Hong, K. H. Han, J. W. Hwang et al., "A case of licorice induced hypokalemic paralysis with rhabdomyolysis," Journal of Korean Endocrine Society, vol. 20, pp. 179-182, 2005.

[7] B. J. Kim, Y. H. Hong, J. J. Sung et al., "A Case of Glycyrrhizin(Licorice)-induced Hypokalemic Myopathy," Journal of the Korean Neurological Association, vol. 24, pp. 389-391, 2006.

[8] S. W. Kwon, H. Y. Jeong, S. K. Yoon, Y. R. Seo, J. Y. Park et al., "A Case of taking self prescribed Licorice Induced Hypokalemic 
Paralysis," Journal of the Korean Oriental Medicine, vol. 32, pp. 134-138, 2011.

[9] H. Korri, A. Awada, W. Baajour, M. Beaini, and W. Nasreddine, "Rapidly progressing quadriparesis secondary to licorice (souss) intoxication," Journal Medical Libanais, vol. 60, no. 2, pp. 117-119, 2012.

[10] M. Shah, C. Williams, A. Aggarwal, and W. M. Choudhry, "Licorice-related rhabdomyolysis: a big price for a sweet tooth," Clinical Nephrology, vol. 77, no. 6, pp. 491-495, 2012.

[11] P. Pant, L. Nadimpalli, M. Singh, and J. C. Cheng, "A case of severe hypokalemic paralysis and hypertension. Licoriceinduced hypokalemic paralysis," The American Journal of Kidney Diseases, vol. 55, no. 6, pp. A35-A37, 2010.

[12] A. C. Meltem, C. Figen, M. A. Nalan et al., "A hypokalemic muscular weakness after licorice ingestion: a case report," Cases Journal, vol. 2, no. 9, article 8053, 2009.

[13] T. Yamamoto, M. Hatanaka, J. Matsuda et al., "Clinical characteristics of five elderly patients with severe hypokalemia induced by glycyrrhizin derivatives," Japanese Journal of Nephrology, vol. 52, no. 1, pp. 80-85, 2010.

[14] C. Templin, M. Westhoff-Bleck, and J.-R. Ghadri, "Hypokalemic paralysis with rhabdomyolysis and arterial hypertension caused by liquorice ingestion," Clinical Research in Cardiology, vol. 98, no. 2, pp. 130-132, 2009.

[15] F. Francini-Pesenti, M. Puato, A. Piccoli, and F. Brocadello, "Liquorice-induced hypokalemia and water retention in the absence of hypertension," Phytotherapy Research, vol. 22, no. 4, pp. 563-565, 2008.

[16] G. C. Isaia, C. Pellissetto, M. Ravazzoli, and C. Tamone, "Acute adrenal crisis and hypercalcemia in a patient assuming high liquorice doses," Minerva Medica, vol. 99, no. 1, pp. 91-94, 2008.

[17] A. E. van den Bosch, J. M. van der Klooster, D. M. H. Zuidgeest, R. J. T. Ouwendijk, and A. Dees, "Severe hypokalaemic paralysis and rhabdomyolysis due to ingestion of liquorice," Netherlands Journal of Medicine, vol. 63, no. 4, pp. 146-148, 2005.

[18] F. J. Gennari, "Hypokalemia," The New England Journal of Medicine, vol. 339, no. 7, pp. 451-458, 1998.

[19] A. Svare, "A patient presenting with symptomatic hypomagnesemia caused by metformin-induced diarrhoea: a case report," Cases Journal, vol. 2, no. 10, article 156, 2009.

[20] H. Yoshikawa, T. Watanabe, and T. Abe, "Tubulo-interstitial nephritis caused by sodium valproate," Brain and Development, vol. 24, no. 2, pp. 102-105, 2002.

[21] D. Widodo, B. Setiawan, K. Chen, L. Nainggolan, and W. D. Santoso, "The prevalence of hypokalemia in hospitalized patients with infectious diseases problem at Cipto Mangunkusumo Hospital, Jakarta," Acta Medica Indonesiana, vol. 38, no. 4, pp. 202-205, 2006. 


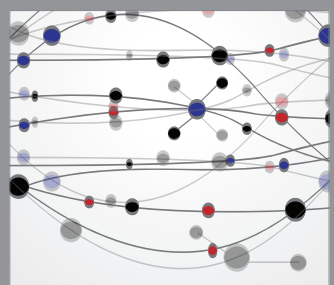

The Scientific World Journal
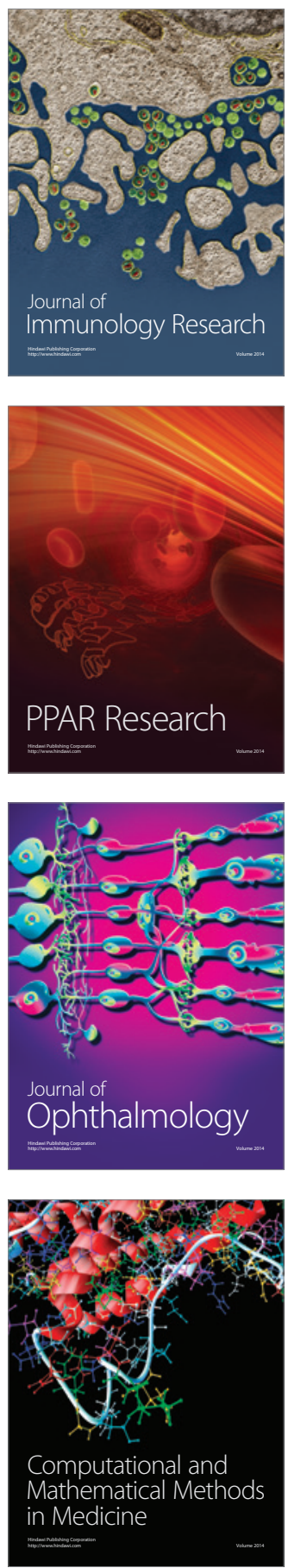

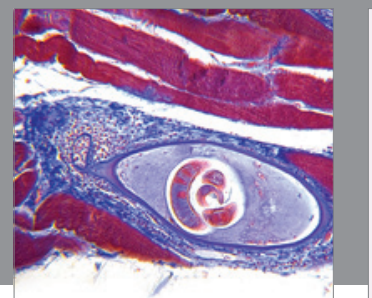

Gastroenterology

Research and Practice
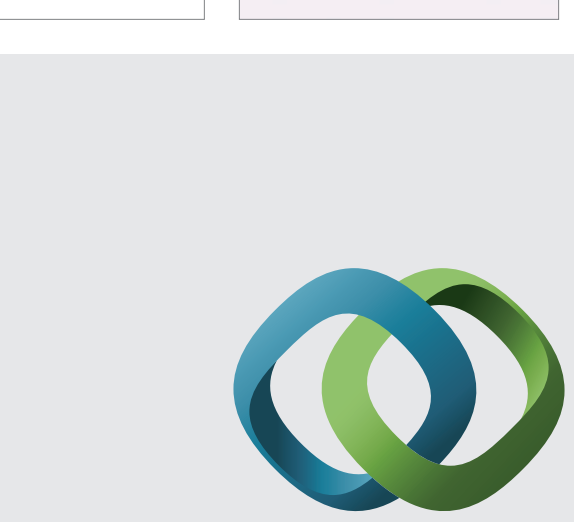

\section{Hindawi}

Submit your manuscripts at

http://www.hindawi.com
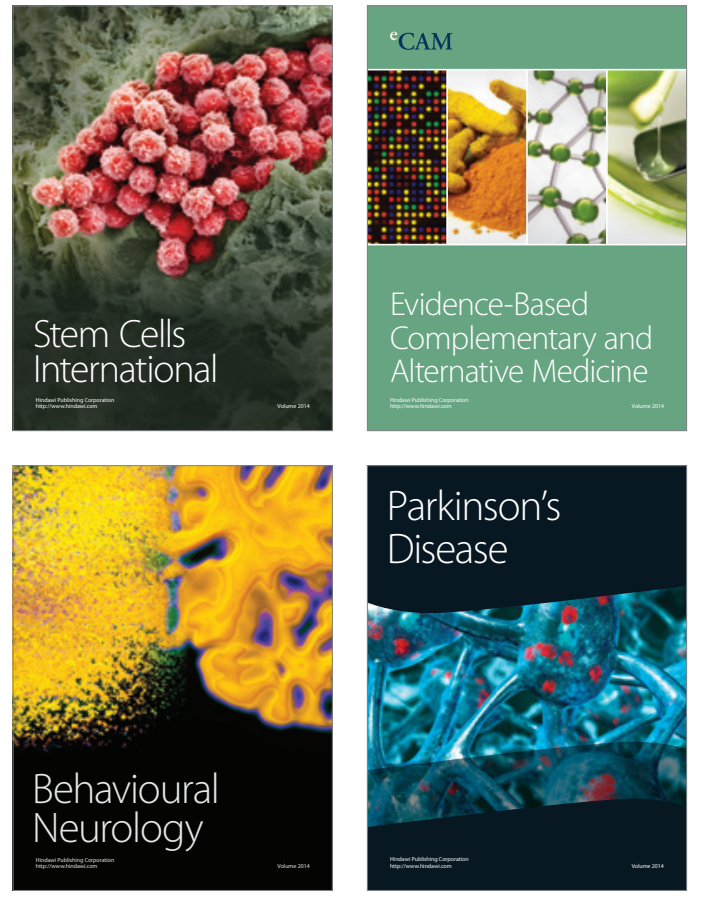
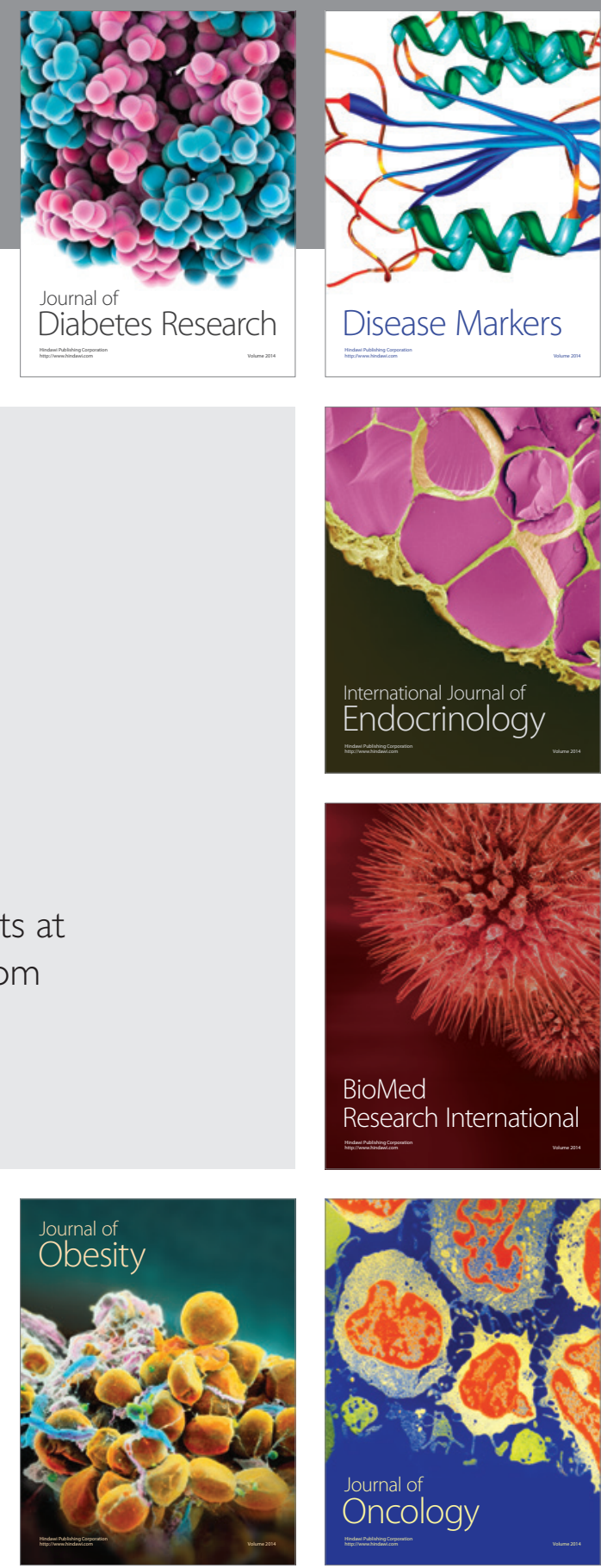

Disease Markers
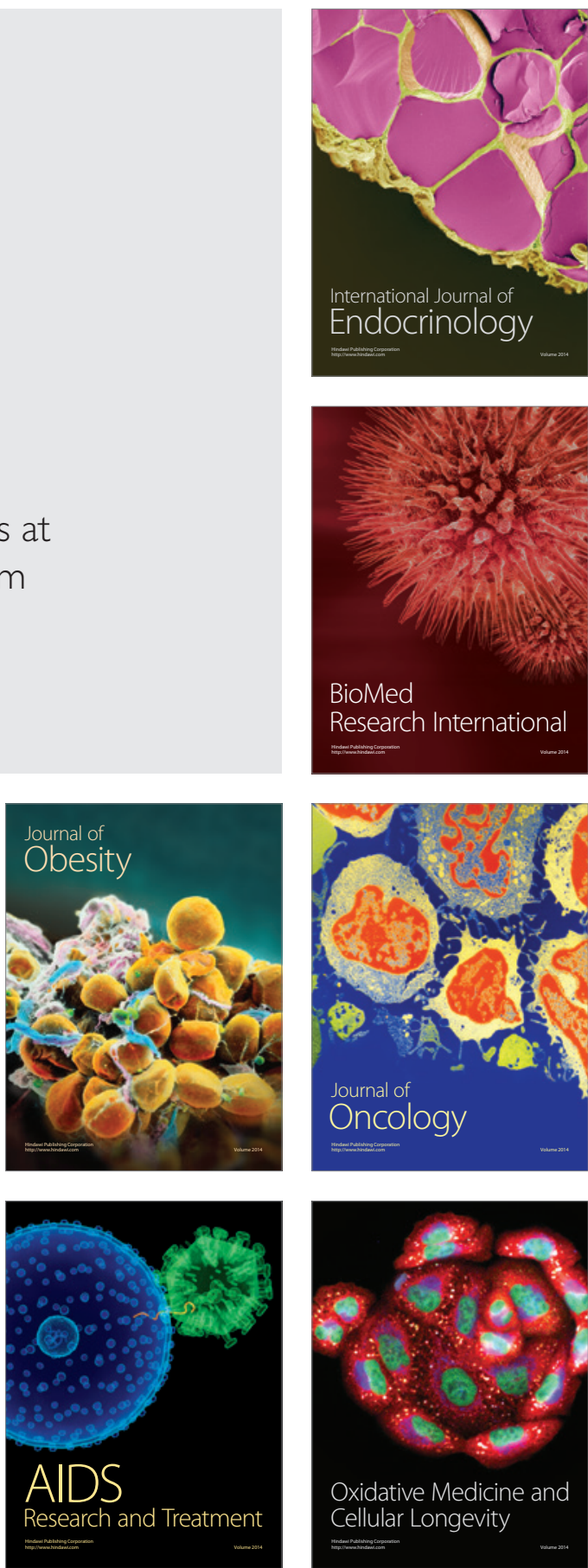